\title{
MATCHING WEAK COUPLING AND QUASICLASSICAL EXPANSIONS FOR DUAL QES PROBLEMS
}

\author{
MICHAEL KAVIC \\ Department of Physics, University of Minnesota Tate Laboratory of Physics, \\ 116 Church Street S.E. Minneapolis, MN 55455, USA
}

\begin{abstract}
Certain quasi-exactly solvable systems exhibit an energy reflection property that relates the energy levels of a potential or of a pair of potentials. We investigate two sister potentials and show the existence of this energy reflection relationship between the two potentials. We establish a relationship between the lowest energy edge in the first potential using the weak coupling expansion and the highest energy level in the sister potential using a WKB approximation carried out to higher order.
\end{abstract}

\section{Introduction}

The discovery of quasi-exactly solvable (QES) spectral problems [1-4] provided new opportunities for exploring such interesting issues as the convergence of perturbative series at high orders or the relationship between weak coupling and quasiclassical (WKB) expansions. [5] Of special importance for the second issue is the energy reflection (ER) symmetry of certain QES potentials [5,6]. It was noted that in certain potentials the QES part of the spectrum is symmetric under $E \leftrightarrow-E$. The transformation rule of the corresponding wave functions is also known.

In this work we will limit ourselves to a particular example of the so-called dual potentials [6]. One-dimensional QES problems are based on $\mathrm{sl}(2)$. This is represented as follows,

$$
H \Rightarrow \sum_{a, b} C_{a b} T^{a} T^{b}+\sum_{a} C_{a} T^{a}
$$

where $C$ 's are constants and $T$ 's are sl(2) generators,

$$
\begin{aligned}
T^{+} & =+2 j \xi-\xi^{2} \frac{d}{d \xi}, \\
T^{0} & =-j+\xi \frac{d}{d \xi} \\
T^{-} & =+\frac{d}{d \xi} .
\end{aligned}
$$

The generators act on polynomials of $\xi$ of degree $2 j$ provided $j$ is a semiinteger number. The dual potentials [6] are such that the two sister potential 
are related by the mapping,

$$
E \rightarrow-E .
$$

Quasi-exact solutions in quantum-mechanics problems depend on the cohomology parameter $(j)$. This parameter determines how much of system's spectral levels are included in the algebraic sector, or, in other words, how large the $2 j \times 2 j$ matrix will be [7].

An interesting limit may be taken, $j \rightarrow \infty$. If $V(x)$ is a potential well, it may become deeper as $j$ is increased. If $j \rightarrow \infty$ the lowest energy level can be calculated perturbatively since the anharmonicity becomes small. This is done by performing an expansion about the minimum point, approximating the potential at the minimum as a slightly perturbed harmonic oscillator.

This expansion leads to an expression for the ground state energy. In the dual potential the energy levels are symmetric with respect to zero. So, the energy reflection relationship can lead to an expression for the highest QES energy level of its sister. This expression may be checked by more traditional means. In particular, a WKB approximation can be applied for highly excited states. Each term is calculated using higher order WKB approximations. The WKB expansion can be confronted with the weak coupling expansion for the ground state of the sister potential. They should match. In Ref [5] a self dual potential was studied in this context. Here I analyze a pair of dual potentials with the aim of establishing the matching.

\section{Lowest Energy Calculation}

Let us investigate a particular example.

$$
\begin{gathered}
V_{1}(x)=\frac{1}{2} \sin ^{2} x+\left(2 j+\frac{1}{2}\right) \cos x, \\
V_{2}(x)=\frac{1}{2} \sinh ^{2} x-\left(2 j+\frac{1}{2}\right) \cosh x .
\end{gathered}
$$

The potentials are plotted in Figures 1 and 2. We start from the weak coupling expansion for $V_{1}(x)$. The first step is to calculate an expression for $E_{0}$. Because the potential is periodic the expression corresponds to the lower edge of the lowest energy band.

Let us parenthetically note that at large $j$ the width of the band can be readily evaluated quasiclassically by calculating the tunneling amplitude from $x=\pi$ to $x=3 \pi$,

$$
S_{\text {tunnel }}=8 \sqrt{2 j+\frac{3}{2}},
$$




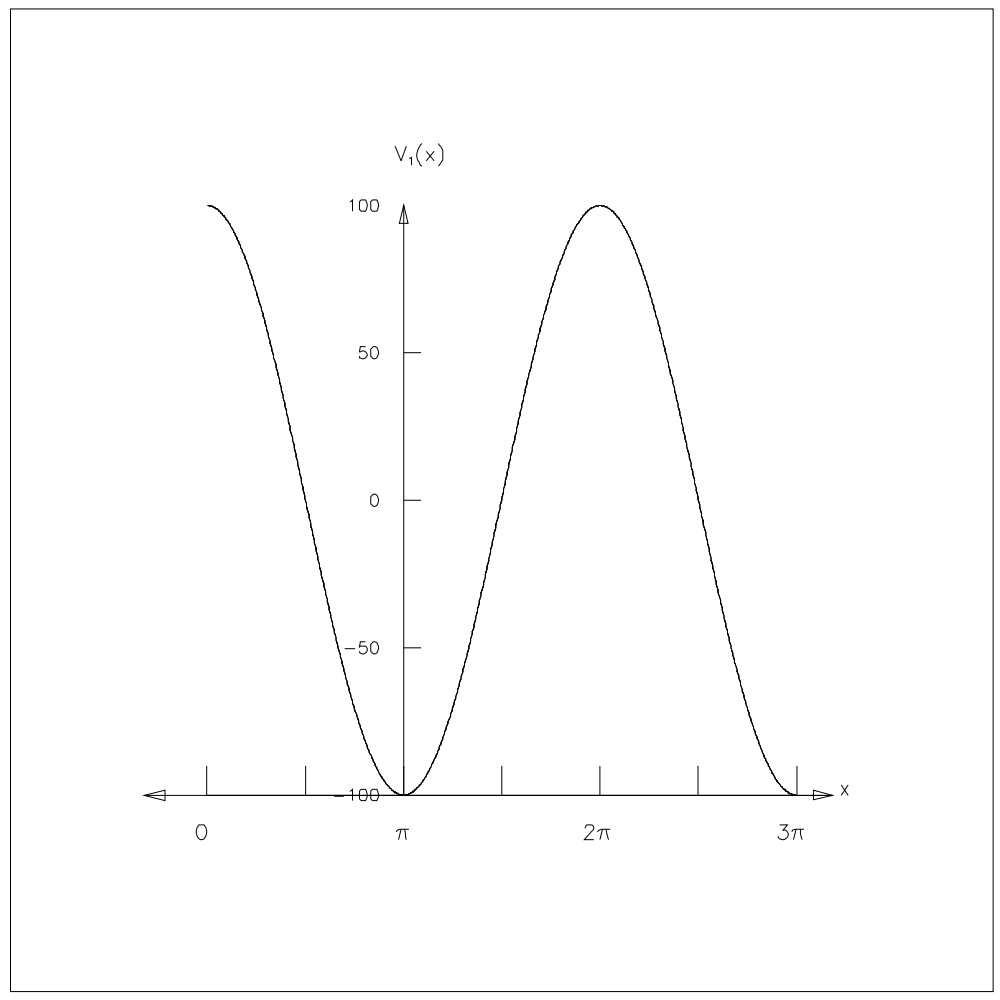

Figure 1: $V_{1}(x), \quad \kappa=101$

and the band width

$$
\delta E \propto e^{-S_{\text {tunnel }}} .
$$

We neglect the effects that arise due to tunneling because they affect only terms exponentially small in $j$ while we address here the power expansion in $\frac{1}{\sqrt{j}}$.

As previously suggested, we consider the limit $j \gg 1$. The eigenvalue problem takes the form

$$
\left[-\frac{1}{2} \frac{d^{2}}{d x^{2}}+\left[\frac{1}{2} \sin ^{2} x+\gamma \cos x\right]\right] \psi(x)=E \psi(x)
$$




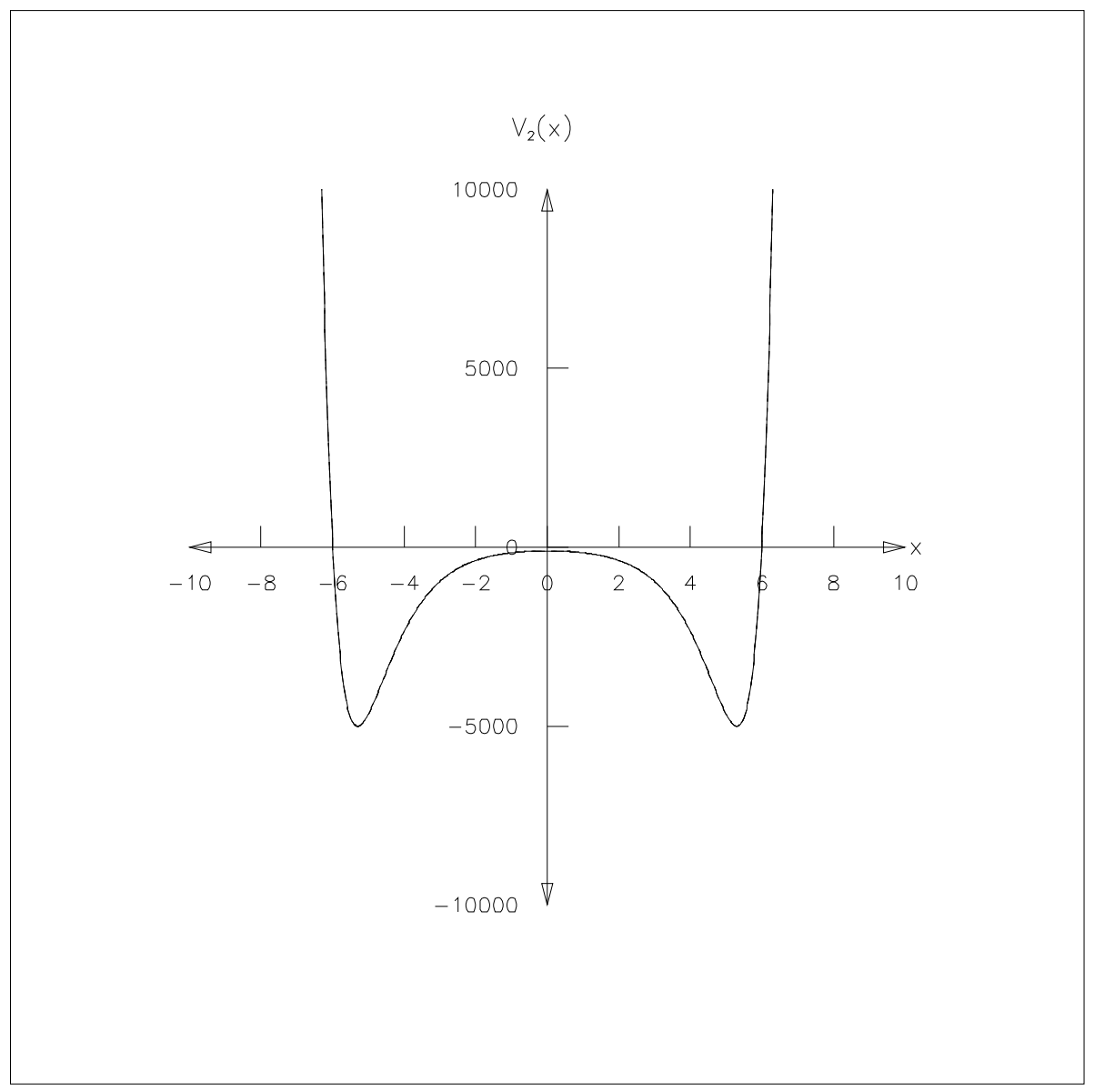

Figure $2: V_{2}(x), \quad \kappa=101$ 
where,

$$
\gamma=2 j+\frac{1}{2}, \quad(j \text { is semi-interger }) .
$$

Taking the limit $\gamma \rightarrow \infty$ causes the potential well to become deeper. The minimum can be calculated to lie at,

$$
x_{\min }=\pi .
$$

Expanding about $\pi$ and using the following notation,

$$
\kappa=(\gamma+1)
$$

gives the following expression for $V_{1}(x)$ :

$V_{1}(x)=-(\kappa-1)+\frac{1}{2} \sqrt{\kappa}(x-\pi)^{2}-\frac{1}{24}(\kappa+3)(x-\pi)^{4}+\frac{1}{720}(\kappa+15)(x-\pi)^{6}+\cdots$,

where the dots denote higher order terms.

The terms of the $\frac{1}{\sqrt{\kappa}}$ expansion can be determined by treating the potential at the minimum as a slightly perturbed harmonic oscillator. The leading term is the classical term,

$$
E_{0}=-(\kappa-1) .
$$

The linear in $(x-\pi)$ term in $V_{1}$ vanishes as do all the odd-power terms. The next term in $E_{0}$ comes from the zero-point oscillation energy for the a harmonic oscillator,

$$
\delta E_{0}=\frac{\omega}{2}=\frac{1}{2} \sqrt{\kappa} .
$$

Next we consider the anharmonic terms. The first order perturbation in $(x-\pi)^{4}$ produces the correction $\propto \kappa^{0}$,

$$
\Delta_{1} E_{0}=-\frac{1}{32} .
$$

The following term is given by adding the second order perturbation of the quartic term,

$$
\Delta_{2} E_{0}=-\frac{7}{64} \frac{1}{\sqrt{\kappa}}
$$

with the first order perturbation of the sextic term,

$$
\widetilde{\Delta_{2} E_{0}}=\frac{1}{384} \frac{1}{\sqrt{\kappa}} \text {. }
$$

Taken together these terms give the following $E_{0}$ expansion:

$$
E_{0}=-\kappa+\frac{1}{2} \sqrt{\kappa}+\frac{31}{32}-\frac{41}{384} \frac{1}{\sqrt{\kappa}} \cdots .
$$




\section{WKB Calculation}

If the energy levels of the sister potentials are symmetric with respect to zero, then multiplying by $(-1)$ the lowest energy expansion should provide an expression for the highest energy level in the algebraic sector of the sister potential. So, the energy reflection property takes the form

$$
-E_{0}=E_{*},
$$

where $E_{*}$ is the highest energy level in the sister potential which is linked to the original potential by mapping $V(x) \rightarrow-V(x i)$. Taking this as the highest even QES energy level in the potential depicted in Fig.2, we determine the number of this (P-even) state in terms of $\gamma$,

$$
n=2\left(\gamma-\frac{1}{2}\right) .
$$

Thus, from our calculation of the lowest energy in $V_{1}$ we have determined an expression for the highest energy in the sister system $V_{2}$. We now use WKB approximations to confirm this expression. Our second potential is

$$
V_{2}(x)=\frac{1}{2} \sinh ^{2} x-\gamma \cosh x .
$$

We scale the energy in the Schrödinger equation such that $\alpha$ is the scaled energy, $E \equiv \gamma \alpha$. We use the convenient change of variable $x=2 t$. Thus,

$$
\left[E-V_{2}(t)\right]=\left(\gamma \alpha-\sinh ^{2} t\right) 2 \cosh ^{2} t .
$$

After scaling the independent variables we obtain the Schrödinger equation,

$$
\epsilon \psi^{\prime \prime}(t)=\left[\left(1-\frac{1}{\gamma} \sinh ^{2} t\right) 2 \cosh ^{2} t-(1-\alpha)\right] \psi(t),
$$

where the small parameter is defined as $\epsilon=\frac{1}{2 \sqrt{2 \gamma}}$. Adopting the convention $\beta=(1-\alpha)$, the scaled energy expansion becomes

$$
\beta=\sum_{n=0}^{\infty} a_{n} \epsilon^{n} .
$$

We will now use WKB approximation carried out to higher order to determine the coefficients of the energy expansion $\left(a_{n}\right)$. The WKB quantization condition $[8,9]$ to $\epsilon^{5}$ is

$$
2\left(\gamma-\frac{1}{2}\right) \pi=\frac{1}{2 \epsilon} \oint d t \sqrt{Q}-\frac{\epsilon}{96} \oint d t \frac{Q^{\prime \prime}}{Q^{3 / 2}}-\frac{\epsilon^{3}}{3072} \oint d t \frac{2 Q^{\prime \prime \prime \prime} Q-7\left(Q^{\prime \prime}\right)^{2}}{Q^{7 / 2}}+\cdots,
$$


in which $Q(t)=2\left[\left(\gamma-\sinh ^{2} t\right) 2 \cosh ^{2} t\right]$ and the contours encircle the two turning points with the branch cut joining them. Once again the dots refer to higher order terms.

Next we expand Eq. (25) in powers of $\epsilon$ and evaluate the contour integrals. In the calculation of each integral we use a second change of variables $y=\sinh t$. The first integral can be done along the real axis. The following integrals need to be evaluated along a contour but can be done in closed form. Each of the integral can be evaluated as a multiple of $\pi$. The factor of $\pi$ is then canceled with the factor of $\pi$ in the quantization condition, leaving a purely algebraic series.

Using the results from Eq. (25) we can calculate the scaled energy coefficients $\left(a_{n}\right)$ in Eq. (24). This results in the following energy expansion

$$
E_{*}=\gamma-\frac{1}{2} \sqrt{\gamma+1}+\frac{1}{32}+\frac{41}{384} \frac{1}{\sqrt{\gamma+1}} \cdots .
$$

Using again the notation $\kappa=\gamma+1$ yields

$$
E_{*}=\kappa-\frac{1}{2} \sqrt{\kappa}-\frac{31}{32}+\frac{41}{384} \frac{1}{\sqrt{\kappa}} \cdots,
$$

in full agreement with the prediction given by the energy reflection relation.

\section{Conclusion}

The $\frac{1}{\sqrt{\kappa}}$ WKB expansion for $V_{2}$ is the same as the weak coupling expansion for $V_{1}$, predicted by the energy reflection relation. Thus we have validated the relation and established the relationship between the energy levels of the two dual potentials. We have shown not only that the relationship between the energy levels exist, but that it persits to higher orders algebraically. This is remarkable because the pertubation calculation and the WKB approximation are independent of one another. Thus, a deeper principle must underlie the symmetry that exists between the energy levels of the two potentials.

\section{Acknowledgements}

M.K. thanks Professor Mikhail Shifman for his careful guidance and generous support, and he thanks Xin Rui Hou, Justin Hietala, and Steven Giron for their shared knowledge and patient assistance.

\section{References}

1. A. V. Turbiner, Commun. Math. Phys. 118, 467 (1988). 
2. A. Ushveridze, Fiz. Elem. Chst. At. Yad, 20, 1185 (1989) [Sov. J. Part. Nucl. 20, 504 (1989)] and earlier works of the author cited therein.

3. M. A. Shifman and A. V. Turbiner, Commun. Math. Phys. 126, 347 (1989).

4. N. Kamran and P. Olver, J. Math Anal. Apl. 145, 342 (1990).

5. M. A. Shifman and A. Turbiner, Phys. Rev. A 59, 1791 (1999) hepth/9806006.

6. A. Krajewska, A. Ushveridze, and A. Walczak, Mod. Phys. Lett. A12, 1225 (1997) hep-th/9601025].

7. For a pedagogical review see in M. A. Shifman, ITEP Lectures in Particle Physics And Field Theory, (World Scientific, Singapore, 1999), Vol. 2, p. 775 .

8. C. M. Bender and S. A. Orszag, Advanced Mathematical Methods for Scientists and Engineers (McGraw-Hill, New York, 1978), Chap. 10.

9. C. M. Bender, G. V. Dunne and M. Moshe, Phys. Rev. A 55, 2625 (1997) arXiv:hep-th/9609193. 